\title{
IBUPROFEN TIMING FOR HAND SURGERY IN AMBULATORY CARE
}

\author{
Enrico Givliani ${ }^{1}$, Anna Blanchil ${ }^{1}$, Augusto Marcuzzi ${ }^{1}$, Antonio LandI $^{1}$, Alberto BarbierI ${ }^{1}$
}

\section{ABSTRACT}

Objective: To evaluate the effect of pre-operative administration of ibuprofen on post-operative pain control vs. early post-operative administration for hand surgery procedures performed under local anaesthesia in ambulatory care. Methods: Candidates to trigger finger release by De Quervain tenosynovitis and carpal tunnel operation under local anesthesia were enrolled in the study. Group A received $400 \mathrm{mg}$ ibuprofen before the operation and placebo after the procedure; group $B$ received placebo before the operation and ibuprofen $400 \mathrm{mg}$ at the end of the procedure; both groups received ibuprofen $400 \mathrm{mg}$ every $6 \mathrm{~h}$ thereafter. Visual analogue scale (VAS) was measured at fixed times before and every 6h after surgery, for a total follow-up of 18h. Results: Groups were similar according to age, gender and type of surgery. Median VAS values did not produce any statistical significance, while there was a statistically significant difference on pre-operative and early post-operative VAS values between groups ( $A-8.53 \mathrm{~mm}$ vs. B $3.36 \mathrm{~mm}, p=0.0085$ ). Conclusion: Average pain levels were well controlled by local anesthesia and post-operative ibuprofen analgesia. Pre-operative ibuprofen administration can contribute to improve early pain management. Level of Evidence II, Therapeutic Studies.

Keywords: Ibuprofen. Analgesics/therapeutic use. De Quervain Disease. Trigger finger disorder. Carpal tunnel syndrome.

Citation: Giuliani E, Bianchi A, Marcuzzi A, Landi A, Barbieri A. Ibuprofen timing for hand surgery in ambulatory care. Acta Ortop Bras. [online]. 2015;23(4):188-91. Available from URL: http://www.scielo.br/aob.

\section{INTRODUCTION}

A large number of out-patient hand surgery operations are performed for treating mildly incapacitating and painful hand pathologies. Such pathologies may arise due to both genetic and environmental risk factors; some of them are, in fact, are work-related. ${ }^{1}$ Some of the most common clinical presentations include: trigger finger, ${ }^{2}$ De Quervain tenosynovitis ${ }^{3}$ and carpal tunnel syndrome. ${ }^{4}$ These pathologies tend to affect individuals in their late adulthood, with the potential to impair both quality of life and ability to work.

Treatment options depend on the type of alteration but can potentially range from splinting (carpal tunnel) ${ }^{5}$ to local steroid injection ${ }^{6}$ to surgery (both open and endoscopic), ${ }^{7}$ which is the treatment of choice in the most severe cases or when all other options have failed.

Trigger finger release, carpal tunnel release, De Quervain tenosynovitis operation, surgical correction of minor bone injuries or pathologies are characterized by similarly low intra- and post-operative pain levels, which can be successfully controlled by local anaesthesia and non-steroidal anti-inflammatory drugs (NSAIDs) respectively.

Anaesthesia for hand surgery largely relies on local or regional techniques ${ }^{8,9}$ that have proven to be safe and effective, especially in the out-patient setting where the management of a general anaesthesia would be more difficult.

An effective post-operative pain control strategy is a prerequisite of any surgical procedure. In the out-patient setting the anesthesiologist needs a protocol that can be easily implemented at home with a low incidence of even mild complications and good tolerability.

NSAIDs are the post-operative pain therapy of choice for their effectiveness to control bone and soft-tissue pain. They can be associated with acetaminophen for the treatment of break-through pain. ${ }^{10}$

A standard therapy at full NSAID dosage should cover the first 18-24 hours after the operation. A local anesthetic activity is present during the early post-operative period with variable duration according to the drug used.

Ibuprofen is a well-tolerated NSAID due to its low gastric toxicity and good anti-inflammatory activity.

The aim of this study is to compare two different ibuprofen post-operative pain management schemes for minor hand surgery procedures performed with local anaesthesia in an outpatient setting in relation to pain control.

All the authors declare that there is no potential conflict of interest referring to this article.

\footnotetext{
1. Policlinico Teaching Hospital, University of Modena and Reggio Emilia, Modena, Italy.
}

Work developed at the Università di Modena e Reggio Emilia Policlinico di Modena, Departmento dell'Apparato Locomotore e Chirurgia della Mano, Modena, Itália. Correspondence: Alberto Barbieri, Unità di Terapia Intensiva, Policlinico di Modena, Via del Pozzo 71, 41124 Modena, Itália. alberto.barbieri@unimore.it 
The main objective of the study is pain level comparison, measured as visual analog scale (VAS) between the treatment groups at scheduled times in the peri-operative period.

Secondary outcomes were VAS value variation before the operation and during follow-up between groups, the use of rescue medication, and the rate of complications related to pain management strategy.

\section{METHODS}

Ethical approval for this phase 4 triple blind randomized trial (Ethical Committee $N^{\circ}$ 5/2009) was provided by the Provincial Ethics Committee of Modena (Comitato Etico Provinciale), Modena, Italy (Chairperson Prof. Sandro Spinsanti) on February 5, 2009.

Patients included in this study were at least 18 years old and candidates for trigger finger release, carpal tunnel release, De Quervain tenosynovitis operation, surgical correction of minor bone injuries or pathologies under local anaesthesia.

Patients were excluded from this study on the basis of allergy to the drug(s) used and a positive medical history of gastric or duodenal ulcers.

The study was conducted at the Policlinico Teaching Hospital (Modena, Italy) in the Department of Pathologies of the Locomotor System. Enrollment for this study covered the period from February 6, 2009 to May 16, 2011.

Each patient was randomly assigned to one group:

Group A: ibuprofen 400 mg 30 minutes before the operation, placebo after the procedure and ibuprofen $400 \mathrm{mg}$ every 6 hours thereafter for a total duration of 18 hours.

Group B: placebo 30 minutes before the operation, ibuprofen $400 \mathrm{mg}$ at the end of the procedure and every 6 hours thereafter for a total duration of 18 hours.

The rescue medication was $1 \mathrm{~g}$ acetaminophen every 6 hours for both groups if reported pain was greater than VAS 40 $\mathrm{mm}^{11}$. Every patient also received a $30 \mathrm{mg}$ lansoprazole capsule prior to the operation.

$A B$, the pharmacist, prepared the randomization sequence using Microsoft Excel ${ }^{\complement}$

$\mathrm{AB}$ prepared placebo and treatment ibuprofen capsules, enclosed them in consecutively numbered sealed envelopes in the specific sequence that each group required. $A B$ then stored envelopes in consecutively numbered sealed boxes, each representing the therapy of one individual patient. No reference to treatment group was present on either envelopes or boxes. $\mathrm{AIB}, \mathrm{VE}, \mathrm{CDE}$, medical students, registered the patients, informed them about the study protocol, explained VAS compilation and handed them VAS record modules and a treatment box following the consecutive sequence generated by AB. Neither the patients nor the experimenters were aware of the treatment group to which they were assigned.

After the attending Anesthesiologist obtained written informed consent, patients were enrolled.

VAS was recorded at fixed time intervals: T0 - pre-operative time, 30 minutes before the operation, $\mathrm{T} 1$ - early post-operative, at the end of the procedure, T2 6, T3 12, T4 18 hours after the operation. The patients were asked to record VAS values on specially designed record sheets by drawing a cross on a straight, 100 $\mathrm{mm}$-long, horizontal, black line and indicate for each time window if they had taken the rescue medication provided.

A separate adverse effect record form was also given to the patients.
A nurse administered topical local anaesthesia using about $2 \mathrm{~g}$ of EMLA ${ }^{\circledR}$ cream $^{12}$ over a surface of roughly $10 \mathrm{~cm}^{2}$ for about 20 minutes before the hand surgeon injected the site of operation with 1-2 mg/kg, of patient lean mass, $2 \%$ mepivacaine hydrochloride solution under the supervision of an anesthesiologist. The nurse, the hand surgeon, and the supervising anesthesiologist were blinded to treatment regimen.

The operations were performed by the same hand surgery team. A tourniquet was used in every procedure to limit blood loss.

VAS records were collected during the first post-operative follow-up visit.

DE entered data onto a computerized Microsoft Excel ${ }^{\odot}$ database. At the end of the study, when enrollment was completed, AB disclosed the patient group assignment without revealing the treatment regimen that each group had received.

EG conducted statistical analysis blind to the treatment regimen. When statistical analysis was complete, $A B$ disclosed the treatment regimen.

\section{Statistical analysis}

Data distribution was tested for normality with a skewness-kurtosis test; normally distributed variables were reported as mean \pm standard deviation (SD); non-normally distributed variables were expressed as medians with range (from minimum to maximum); and dichotomous variables were expressed as percentages. Normally distributed variables were compared by using the Student's t-test; non-normally distributed variables were compared with the Mann-Whitney test; non-normally distributed paired variables were compared by the Wilcoxon signed rank test; percentages were compared by using $\chi^{2}$ test; $p$-values $<0.05$ were considered statistically significant.

\section{RESULTS}

A total of 100 patients were enrolled from February 6, 2009 to May 16,2011 . Thirteen were lost during follow-up due to patient failure to complete VAS records, 87 completed the observation period (43 in group A, 44 in group B). Figure 1 shows the enrollment flowchart.

Demographic variables are summarized in Table 1. After randomization, grups were similar regarding age, sex and type of surgery, no statistically significant difference was found for these variables. The duration of all procedures was under 45 minutes. No anaesthesia-related complications were reported during the procedures. In no case it was necessary to modify the anesthetic regimen because of inadequate pain control.

Median VAS values according to treatment group are reported in Figure 2. No statistically significant difference was found. VAS value variation between T0 and T1, T1 and T2, T2 and T3, T3 and T4 was then calculated, a skewness-kurtosis test for normality was negative, so a Mann-Whitney test was used to compare VAS values median difference according to treatment group. (Figure 3) A statistically significant difference was found between T0$\mathrm{T} 1$ between group $\mathrm{A}$ and $\mathrm{B}$. Table 2 reports the median VAS values variation: group A presented a lower variation than group B at T0-T1. A non-parametric K-sample equality of median test was performed to assess the difference in pain levels related to the type of surgery. No statistically significant differences were present during the procedures and follow-up.

There was no statistically significant difference in the use of 


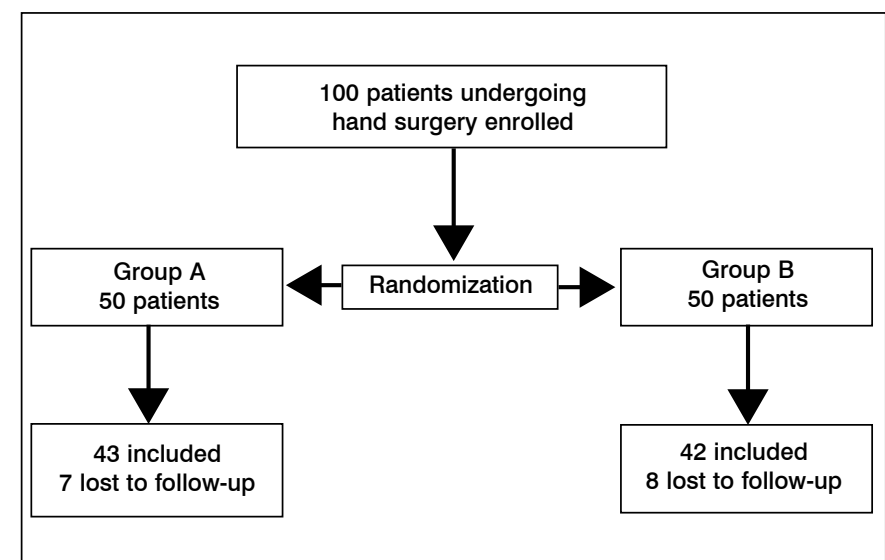

Figure 1. Enrolment flowchart.

Table 1. Demographic variables of the studied groups. Average age \pm standard deviation (SD), sex as percentage of males, type of surgery as percentage of the total number of procedures for each group. No statistically significant differences were reported (all p-values $>0.05$ ).

\begin{tabular}{c|c|c}
\hline Parameter & $\begin{array}{c}\text { Group A } \\
\text { Mean SD }\end{array}$ & $\begin{array}{c}\text { Group B } \\
\text { Mean SD }\end{array}$ \\
\hline Age (years) & 52.3413 .87 & 53.169 .36 \\
\hline Sex (\% of males) & Percentage & Percentage \\
\hline Type of surgery & $32.55 \%$ & $27.27 \%$ \\
\hline Trigger finger & & \\
\hline Carpal tunnel & $42.86 \%$ & $30.23 \%$ \\
\hline De Quervain tenosynovitis & $4.76 \%$ & $11.63 \%$ \\
\hline Minor bone injuries & $38.10 \%$ & $46.51 \%$ \\
\hline
\end{tabular}

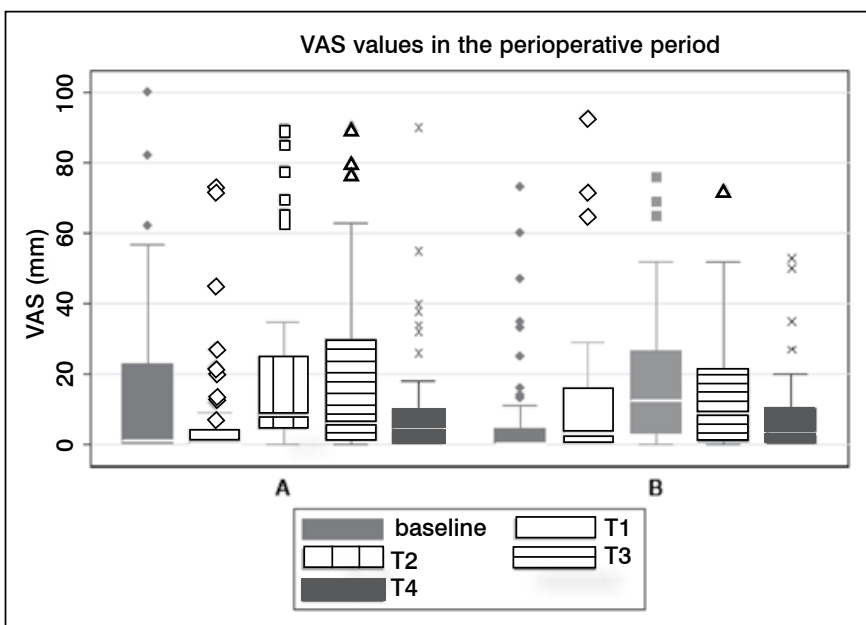

Figure 2. Median VAS values ( $\mathrm{mm}$ ) at baseline (T0), after the procedure (T1), at 6 hours (T2), at 12 hours (T3), at 18 hours (T4) after the operation.

rescue therapy during follow-up. Acetaminophen was used 6 times in group A and 6 times in group B.

No drug-related adverse effects were reported during follow-up. There was a main limitation to these results: the relatively small sample size, which may reduce the clinical significance of these findings, which may be further magnified by the fact that data were collected in a single centre, which may lead to a selection bias on the basis of a specific case-mix treated during data collection.
Table 2. Median VAS values $(\mathrm{mm})$ differences between $\mathrm{T} 0$ and $\mathrm{T} 1, \mathrm{~T} 1$ and T2, T2 and T3, T3 and T4. T0, 30 minutes before anesthesia; T1 6 hours after the procedure; T2, at 6 hours; T3, at 12 hours; T4, at 18 hours after the operation.

\begin{tabular}{c|c|c|c}
\hline VAS (mm) & $\begin{array}{c}\text { Group A } \\
\text { Median }\end{array}$ & $\begin{array}{c}\text { Group B } \\
\text { Median }\end{array}$ & p-value \\
\hline T0-T1 & -8.53 & 3.36 & $0.0085^{*}$ \\
\hline T1-T2 & 13.04 & 6.66 & 0.0893 \\
\hline T2-T3 & -1.72 & -3.84 & 0.8575 \\
\hline T3-T4 & -8.05 & -6.11 & 0.9281 \\
\hline
\end{tabular}

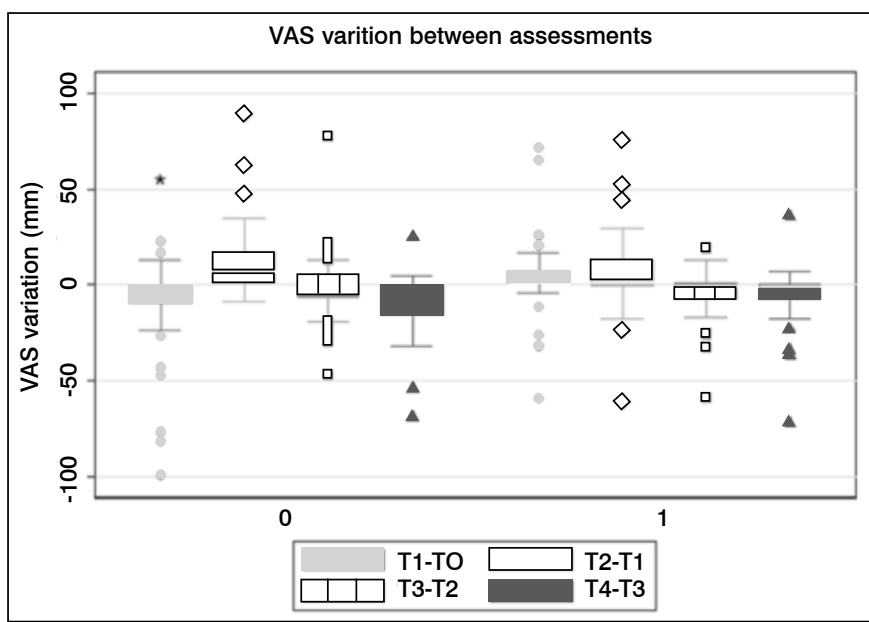

Figure 3. Median VAS values $(\mathrm{mm})$ differences between $\mathrm{T} 0$ and $\mathrm{T} 1, \mathrm{~T} 1$ and T2, T2 and T3, T3 and T4. T0, baseline; T1 after the procedure; T2, at 6 hours; T3, at 12 hours; T4, at 18 hours after the operation.

\section{DISCUSSION}

This triple blind randomized control trial showed a substantial equivalence in post-operative pain control, especially during the later phases of follow-up. Median pain levels were under the threshold for rescue medication for most of the patients.

For minor hand surgery procedures such as trigger finger release, De Quervain tenosynovitis operation topical and local anaesthesia seemed to be a viable solution, especially in the out-patient setting for its safety and effectiveness, an effective postoperative pain control therapy maximizes the benefits of topical and local anaesthesia. Ibuprofen with acetaminophen as rescue therapy offered good post-operative pain relief with a similar VAS values profile between groups.

A pre-operative dose of ibuprofen did not appear to influence post-operative pain levels apart from T1. This, however, may not be considered a pre-emptive analgesic effect, as a way to provide inflammatory mediator reduction by inhibiting their production pathway before its activation. It may be the result of a timely plasma concentration peak to cover the end of such procedures. Ibuprofen is a commonly used NSAID ${ }^{13}$ due to its analgesic properties with a low incidence of the most common adverse effects of this class of drugs. It is well absorbed orally with a peak serum concentrations in 1 to 2 hours after administration. This could be, nonetheless, a useful technique for maintaining a more stable pain curve while the patient is recovering from anaesthesia.

The absence of post-operative complications is in line with out-patient requirements. 
Quality of life was minimally affected as most of the subjects experienced no to mild pain during follow-up. Only a minority of patients reported VAS greater than $40 \mathrm{~mm}$.

\section{CONCLUSIONS}

If administered pre-operatively, ibuprofen can reduce pain levels in the immediate post-operative period, more significantly than an early post-operative dose.
This strategy could better couple anaesthesia and pain control therapy without additional negative side effects.

\section{AKNOWLDGEMENTS/}

The authors wish to acknowledge Pre-scatto Investigators Claudio Castagnetti MD, Alessandro Bonito Lt., Vincenzo Esperti Lt., Concetta D. Evangelista Lt., Marco Venturelli RN for their precious contribution in collecting data for the study.

\section{REFERENCES}

1. Andréu JL, Otón T, Silva-Fernández L, Sanz J. Hand pain other than carpal tunnel syndrome (CTS): the role of occupational factors. Best Pract Res Clin Rheumatol. 2011;25(1):31-42.

2. Ryzewicz M, Wolf JM. Trigger digits: principles, management, and complications. J Hand Surg Am. 2006;31(1):135-46.

3. Ilyas AM, Ast M, Schaffer AA, Thoder J. De quervain tenosynovitis of the wrist. J Am Acad Orthop Surg. 2007;15(12):757-64.

4. Palmer KT. Carpal tunnel syndrome: the role of occupational factors. Best Pract Res Clin Rheumatol. 2011;25(1):15-29.

5. De Angelis MV, Pierfelice F, Di Giovanni P, Staniscia T, Uncini A. Efficacy of a soft hand brace and a wrist splint for carpal tunnel syndrome: a randomized controlled study. Acta Neurol Scand. 2009;119(1):68-74.

6. Peters-Veluthamaningal C, van der Windt DA, Winters JC, Meyboom-de Jong B. Corticosteroid injection for trigger finger in adults. Cochrane Database Syst Rev. 2009;(1):CD005617.

7. Huisstede BM, Randsdorp MS, Coert JH, Glerum S, van Middelkoop M, Koes BW. Carpal tunnel syndrome. Part II: effectiveness of surgical treatments-a systematic review. Arch Phys Med Rehabil. 2010;91(7):1005-24.
8. Castellanos J, Ramírez C, De Sena L, Bertrán C. Transthecal digital block: digital anaesthesia through the sheath of the flexor tendon. J Bone Joint Surg Br. 2000;82(6):889.

9. Macaire P, Choquet O, Jochum D, Travers V, Capdevila X. Nerve blocks at the wrist for carpal tunnel release revisited: the use of sensory-nerve and motor-nerve stimulation techniques. Reg Anesth Pain Med. 2005;30(6):536-40.

10. Daniels SE, Goulder MA, Aspley S, Reader S. A randomised, five-parallel-group, placebo-controlled trial comparing the efficacy and tolerability of analgesic combinations including a novel single-tablet combination of ibuprofen/ paracetamol for postoperative dental pain. Pain. 2011;152(3):632-42.

11. Turan $A$, Memiş $D$, Karamanlioğlu $B$, Güler $T$, Pamukçu Z. Intravenous regional anesthesia using lidocaine and magnesium. Anesth Analg. 2005;100(4):1189-92.

12. Agarwal A, Yadav G, Gupta D, Tandon M, Dhiraaj S, Singh PK. Comparative evaluation of myolaxin and EMLA cream for attenuation of venous cannulation pain: a prospective, randomised, double blind study. Anaesth Intensive Care. 2007;35(5):726-9.

13. Al-Homrany MA1, Irshaid YM. Pharmacoepidemiological study of prescription pattern of analgesics, antipyretics, and nonsteroidal anti-inflammatory drugs at a tertiary health care center. Saudi Med J. 2007;28(3):369-74. 